\title{
Les « biographies » d'une icône volante et le statut de quelques récits dans une région balkanique
}

"Biographies" of a Flying Icon and the Status of a Few Narratives in a Balkan Region

\section{Galia Valtchinova}

\section{CpenEdition}

Journals

Édition électronique

URL : https://journals.openedition.org/clo/5719

DOI : $10.4000 /$ clo.5719

ISSN : 2266-1816

\section{Éditeur}

INALCO

Édition imprimée

Date de publication : 18 juin 2019

Pagination : 25-54

ISBN : 978-2-85831-348-8

ISSN : 0396-891X

\section{Référence électronique}

Galia Valtchinova, "Les «biographies » d'une icône volante et le statut de quelques récits dans une région balkanique », Cahiers de littérature orale [En ligne], 85 | 2019, mis en ligne le 19 juin 2020, consulté le 22 mars 2022. URL : http://journals.openedition.org/clo/5719 ; DOI : https://doi.org/ $10.4000 /$ clo.5719

\section{(c) (1) (\$)}

Cahiers de littérature orale est mis à disposition selon les termes de la Licence Creative Commons Attribution - Pas d'Utilisation Commerciale 4.0 International. 


\title{
Les « biographies »d'une icône volante et le statut de quelques récits dans une région balkanique ${ }^{1}$
}

\author{
Galia Valtchinova \\ Université de Toulouse II Jean Jaurès - LISST-CAS, UMR 5193
}

\begin{abstract}
— Elle est vivante, la Sainte Mère-de-Dieu [Sveta Bogorojca $]^{2}$ ! Elle peut éprouver des sentiments et se mettre en colère, et c'est ce qui s'est passé il y a longtemps, quand Elle a quitté Son église située là-bas [geste de la main pour montrer vers la lisière du village] où fut l'ancienne église, il y a les ruines [...]. Elle s'est mise en colère après une femme qui voulut se marier avec un Turc [...] et a décidé d'aller ailleurs, d'abandonner l'église et le village entier. Elle s'est envolée... et ainsi est allée dans le village de Konče. Et depuis ce temps Elle réside dans la vieille église de Konče...
\end{abstract}

1. Reconnaissance. Une première réflexion sur ce cas a été ébauchée dans l'article « Icône et identité : usages sociaux de l'image sacrée chez les Slaves orthodoxes des Balkans » in Anstett E. (dir.), 2009, Un patrimoine sous influences. Usages politiques, religieux et identitaires de l'image dans le monde slave, Petra, Paris, p. 17-35. Le texte qui suit est le fruit d'une longue maturation dont les étapes sont retracées en gardant intentionnellement la note personnelle et le « je » ethnographique. À des moment différents cette maturation a bénéficié d'échanges avec Élisabeth Claverie, Emma Aubin-Boltanski et Charles Stewart, à qui j'exprime ma reconnaissance. Mes remerciements vont également aux deux lecteurs anonymes de la revue Cahiers de littérature orale pour leurs remarques et suggestions.

2. La Vierge est connue en milieu gréco-orthodoxe sous l'appellation de « Mère de Dieu »-Theotokos ou Sveta Bogorodica (ici Bogorojca); plus loin, ce terme est utilisé de manière interchangeable avec les termes français. 
GV : - Comment est-elle allée là-bas, dans son corps [telom] ?

— Oui... c'est Son icône qui y est allée. Son icône est lourde, elle pèse comme un corps - mais elle a bougé et s'est déplacée en volant, car Elle a été très en colère...

Entretien avec M. (80 ans), le 8 août 1999

Cette vignette extraite de mes carnets de terrain restitue le début de l'échange que j'ai eu avec la voyante [vidovita] M. de Bansko, village en République de Macédoine (devenue depuis Macédoine du Nord), la première fois que je l'ai rencontrée. En guise de présentation, elle a produit le récit de ce que j'avais pris

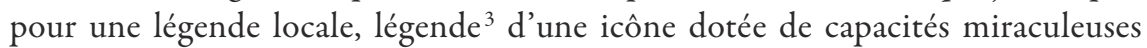
dont celle de se déplacer dans les airs. Ce n'est que plus tard que j'ai pris la mesure du statut spécial de ce récit pour la voyante ainsi que pour les chrétiens orthodoxes du village et au-delà de lui, pour toute une communauté de croyants et non-croyants fonctionnant en réseau ${ }^{4}$, et pu reconstituer les fils reliant ce récit à ce qui s'est avéré être une légende migratoire 5 .

3. Ici, je m'en tiens à la définition de la légende comme « récit historique [qui] met en scène des personnages censés avoir existé, en des lieux dont le nom attesterait de l'ancienneté et de la vérité des faits racontés » (BRU, 1999, § 6). À la différence du récit fictionnel, la légende est «support de croyance et de réalité pseudo-historique ».

4. Après cette première rencontre, je suis retournée à Bansko pour des séjours très courts (ne dépassant pas une semaine) tous les étés jusqu'en 2003, ensuite en 2006, 2007, 2010 et finalement en 2013. La plupart de ces visites eurent comme point d'ancrage le domicile familial de M. et évoluèrent au gré des rencontres avec les membres de sa famille (spécialement son mari et son fils cadet habitant la maison parentale avec sa propre famille) et des proches. M'accompagnant dans mes déplacements, ces derniers m'introduisirent auprès d'autres habitants locaux dans le quartier chrétien-orthodoxe de ce village mixte, ainsi qu'au vaste réseau de villageois et citadins (de Strumica) qui s'était tissé autour de M. pendant près de quatre décennies.

5.J'utilise le concept de «légende migratoire », proposé par Reidar Christiansen (1958) pour désigner des récits que leurs transmetteurs tiennent pour vrais, au moins en partie, mais dont on retrouve ailleurs des variantes soi-disant vécues par d'autres personnages (définition synthétique suggérée par Josiane Bru que je remercie pour son aide). Il est préféré à la catégorisation plus ancienne des légendes en « mémorats » et « fabulats » laquelle, comme D. Hopkin (2010, p. 4) l’a observé, soulève la très importante question du rapport à la mémoire et au vécu personnel, deux paramètres dont Maurice BLOCH (1994) a démontré l'enchevêtrement dans la construction de la mémoire 
L'extrait introduit les deux questions soulevées dans cet article : que signifie l'agir autonome de l'image religieuse et quel est le statut des récits circulant au sein d'une culture balkanique qui donnent à voir une situation extraordinaire, un « miracle » ? La première est abordée en associant la notion d'agentivité, celle d'objet-personne 6 (à laquelle renvoie le E majuscule d'Elle pour parler de l'icône) et celle de « biographie d'objet ${ }^{7}$ », en l'occurrence d'une image sacrée : l'icône dans le christianisme orthodoxe. La recherche anthropologique a bien démontré que l'icône fournit un langage symbolique pour exprimer des relations ou des positions sociales et des registres identitaires de proximité (Herzfeld, 1990 ; Kenna 1985), à la différence de ce qu'enseigne la théologie de l'icône (Hanganu, 2010, p. 45-46) ${ }^{8}$. D’un autre côté, l'idée a été développée (Stewart, 2008 ; 2012) que l'icône serait un medium pour exprimer le fonctionnement d'ordres historiques différents, un objet focalisant l'imagination historique d'une société, ce qui complexifie davantage l'agentivité de cette image sacrée.

L'autre interrogation porte sur le statut de récits présentant certaines caractéristiques de la légende religieuse sur le plan formel ; examinés de près dans leurs contextes d'énonciation, ils s'avèrent cependant imbriqués dans des conglomérats de créations orales faits de récits de vie, de réactualisation de motifs migratoires et d'adaptations locales d'énoncés historiques. En scrutant ces récits dans leurs contextes et l'acte de narration lui-même, on s'attachera à saisir la capacité des acteurs à exprimer, à travers des histoires d'icônes, leurs idées d'enracinement spatio-temporel ainsi que leur appartenances (ou résistances)

historique. Le rapport à la mémoire, au vécu personnel et à la « vérité » demeure central dans notre cas, occultant le lien du récit à un motif migratoire.

6. Pour l'objet-personne ou objet disséminé, termes associés à la traduction française (2009) de l'ouvrage d'A. GELL (1998) qui marque un tournant dans l'approche anthropologique de l'agentivité (cf. Allard, 2010), je m'en tiens à l'usage qu'en fait Aubin-Boltanski (2012a, 2012b) dont les ethnographies émanent de contextes proches.

7. La notion de biographie d'objet remonte à celle de «vie sociale des objets » (Appadurai, 1986), en particulier Kopytoff, 1986 ; pour la « biographie d'icône » comme un enchevêtrement de biographie personnelle et matérielle, cf. HANGANU, 2010.

8. Dans la théologie orthodoxe, l'icône est la représentation de la figure divine (Dieu, le Christ, la Vierge, les saints) et ne tire sa force et/ou ses pouvoirs que du fait de représenter, de servir de support matériel qui conduit la grâce divine. Dans la pratique cependant, la tendance générale est à vénérer les icônes aussi bien pour l'entité représentée qu'en ellesmêmes, en tant que sources de grâce divine. C'est cette tendance que tente de combattre l'iconoclasme à Byzance : comme le démontre Marie-José Mondzain (1996), derrière cette querelle des images il s'agit bien d'une « économie de l'image sacrée » et du principe divin tout court. 
identitaires ${ }^{9}$. Avant d'aller au cœur du sujet, voyons le contexte de l'enquête, les acteurs et des lieux.

\section{Les récits de M., femme visionnaire dans un village macédonien}

Le premier de la série de récits examinés (dont est tiré la vignette) fut produit en public un 8 août, en présence de l'ethnographe et d'une demi-douzaine de curieux, dans le contexte de la fête de la sainte patronne du village de Bansko. Situé à l'extrémité Sud-Est de la République de Macédoine du Nord, le village de Bansko est l'un des plus grands et prospères dans le district frontalier (par rapport à la Grèce et à la Bulgarie) de Strumica, et lui-même très proche des deux frontières. Deux communautés religieuses y habitent en quartiers séparés désignés par le terme de mahalles (mot renvoyant aux réalités de l'époque ottomane qui, localement, se termine en 1912), visiblement en bonne entente : les brefs échanges que j'engage en traversant le village pour aller à l'église montrent que tout le monde est bien informé de la fête en cours. La cohabitation religieuse semble réussie et stable : visitant les lieux dans les années 1970, l’ethno-démographe Jovan Trifunoski, à qui l'on doit la seule ethnographie du village, insiste sur la mixité religieuse et observe des pratiques syncrétiques au turbé [mausolée d'un saint musulman hétérodoxe] ainsi qu'à l'église ${ }^{10}$. Chaque mahalle est dominé par le lieu de culte : l'église blottie dans les arbres du piémont, la mosquée active en contrebas de la rue principale ainsi que le minaret semi-détruit d'une plus ancienne mosquée dans la plaine. D'autres lieux de culte musulmans sont visibles à l'entrée du village où, près des bains ottomans, deux bâtisses semi-ruinées indiquent l'emplacement d'un ancien tekké $e^{11}$ et d'une école coranique ${ }^{12}$. Les différences ethniques semblent

9. Dans cet axe, ce texte a bénéficié de la lecture de l'introduction ainsi que plusieurs contributions dans Clerc-Renaud \& Leguy, 2016, en particulier Neveu, 2016 et ISNART, 2016.

10. Une majorité turque est attestée pour le village à la fin du XIX ${ }^{\mathrm{e}}$ siècle : voir KANČov, 1996, p. 159. Cf. Trifunoski, 1979, p. 90, 101, etc. Réalisée dans le cadre d'un vaste projet de monographies villageoises couvrant l'ensemble de l'ancienne Yougoslavie fédérative, cette dernière étude est exemplaire de l'approche anthropo-géographique dominante dans l'ethnologie yougoslave jusqu'aux années 1960.

11. Souvent traduit comme « monastère » ou « loge de derviches », ce terme - synonyme de zawiya - s'applique au lieu de réunion de confréries musulmanes hétérodoxes : voir Clayer, 2000.

12. Si l'aménagement des sources thermales date du passé ottoman, une volonté de faire remonter l'histoire du village à l'époque pré-ottomane a conduit à des fouilles 
effacées par l'appartenance religieuse : les chrétiens-orthodoxes s'identifient comme des Macédoniens et les musulmans comme des Turcs ; on s'aperçoit toutefois que les Roms constituent un segment conséquent dans les deux groupes ${ }^{13}$.

Ce qui se présentait comme la fête du village était celle de la communauté chrétienne : le jour de commémoration d'une sainte orthodoxe, sainte Parascève ou Petka, martyre romaine, la sainte la plus vénérée par les différentes communautés orthodoxes des Balkans ${ }^{14}$ après (et parfois à égalité avec) la Vierge. Contrairement aux attentes de l'ethnographe habituée aux réalités balkaniques, ce n'était pas la patronne de la paroisse : la fête se déroulait autour d'une petite chapelle sise près de l'église paroissiale et dédiée à ladite sainte. C'était la construction plus élaborée de la série de minuscules chapelles et captages d'eau «bénite » [ sveta voda ${ }^{15}$ qui formaient une constellation autour de l'église visiblement récente (des années 1940). Comme je l'appris par la suite, elles furent construites à la suite de révélations divines données à $M$. pendant plusieurs années, après l'établissement du couple formé avec son mari à proximité de la localité, dans les années 1950. Native d'un village voisin, cette femme mariée au fils d'un Valaque réfugié de

archéologiques qui ont mis au jour une importante construction thermale de l'époque romaine. Selon TRIfUNOSKI (1979, p. 10), Bansko possédait deux tekkés dont celui sis à proximité de la source thermale fut un centre cultuel important.

13. Les Roms, ici traditionnellement appelés Gjupci (nom dérivé d'Égyptiens) comptent pour une bonne part de la population locale; cependant le groupe n'est pas homogène. Le partage entre Roms musulmans et orthodoxes tient compte de l'appartenance religieuse, de la langue parlée et de leurs propres revendications identitaires : cf. TRIFUNOSKI, 1979, p. 14, 59, qui insiste sur leur « mimétisme ethnique ».

14.J'ai étudié ailleurs (en dernier lieu Valtchinova, 2019, p. 19-76) différents aspects du culte et des représentations de « sainte Petka », figure composite dans laquelle se mêlent les hagiographies de trois saintes homonymes. Des nombreuses études sur les aspects folkloriques du culte de cette sainte je retiens Detelić, 2010 ; Mesnil, Popova, 1993.

15. L'invention (dans le sens de découverte - indiqué plus loin par l'usage de l'italique) d'une source d'eau est une action très valorisée sur le plan religieux dans l'islam : selon les hadiths, « donner à boire à celui qui a soif » est l'aumône la plus récompensée. Les captages d'eau sous la forme de fontaines aménagées, à l'usage public, marquent le paysage dans les campagnes balkaniques dont la population est majoritairement musulmane. Il ne faut pas sous-estimer l'aspect pragmatique pour autant : nécessité vitale, l'accès à l'eau est très réglementé dans la campagne et Bansko fait partie des villages où le système traditionnel de gestion de l'eau repose sur une alternance des familles à bénéficier des ressources. La supervision de ce système fut confiée à un notable et pouvait être transmise de père en fils (TRIFUnOSKI, 1979, p. 23). 
la Grèce ${ }^{16}$ détient un immense savoir - qui procède du souvenir - concernant les familles locales et celles des villages voisins, ainsi que le passé de nombreuses communautés villageoises : mémoire autobiographique et mémoire historique se mêlent et interagissent, à la manière décrite par Maurice Bloch (1995), pour remonter au passé éloigné.

La fête, lors de laquelle les locaux viennent lui témoignent le respect, est une occasion pour partager ce savoir-et-mémoire : consciente de son rôle, la voyante narre le passé du village en soulignant le rôle de sa (belle-)famille dans la consolidation de la communauté chrétienne de Bansko ${ }^{17}$. Le discours de M. est un mélange de récits du passé local relevant du souvenir, avec quelques apports de culture historique scolaire, imbriqués dans la narration de ses visions et révélations (le plus souvent oniriques) auxquelles s'agrègent des récits de miracles, localisés dans un ailleurs et avant indéterminé. Tout cela coexiste à l'intérieur d'une culture religieuse de l'écrit passant par l'oreille - une culture aurale ${ }^{18}$ plutôt qu'orale tissée de citations des Écritures, d'hagiographie, de prophéties. Les narrations de la voyante apparaissent ainsi comme des produits complexes résultant de la superposition de plusieurs « sources » entre mémoire et histoire, qui se transmettent essentiellement à l'oral et par l'entendre-lire. Leur survie est assurée par la notoriété et les réseaux personnels de sa famille, mais aussi par un vaste cercle de croyants, deux relais assurant la diffusion de cette « histoire populaire » imbue de religiosité bien au-delà de la communauté orthodoxe locale.

La fête annuelle célébrée le 8 août permet de mettre en avant l'histoire de l'invention du « petit monastère » érigé en 1968, comme l'annonce l'inscription au-dessus de l'entrée, qui serait consécutive à une série de crises visionnaires de M., « asservie » [zarobena $]$ par sainte Petka qu'elle tenait pour sainte tutélaire depuis son enfance. C'est la première et la plus importante de plusieurs structures

16. Ici et plus loin, le terme de réfugié(s) désigne les populations déplacées (de force ou par la force d'un accord) dans la logique d'échange de population pratiqués entre pays du Sud-Est européen à l'issue de la Première Guerre mondiale, avec l'attribution aux déplacés d'un statut de « réfugiés » reconnu par la Société des Nations dès 1922. L'étude anthropologique phare de ces mouvements migratoires est HIRSCHON (1989), conduite en contexte urbain ; l'impact de ces mouvements migratoires en contexte rural est très peu étudié. Voir infra et notes 37,38 et 40 .

17. On trouvera un développement de ces observations dans VAltchinova, 2006, p. 111-136.

18. Dérivé du mot latin pour oreille [auriculum], le terme aural nuance la perception et la communication qui s'effectue par l'oreille et l'ouie. Je retiens la mise au point par Couroucli, 1995 et Gokalp, 1995. 
nées dans les années 1960-1970. À l'intérieur, l'image d'une sainte Parascève à l'iconographie plutôt rare attire l'attention : l'intérêt qu'on lui porte suscite immédiatement un récit de sa découverte : peinte sur toile (matériau rarissime pour les icônes orthodoxes), elle aurait été enroulée et « cachée », puis « oubliée » dans une dépendance de l'église toute proche, avant de se manifester à la voyante en chantant comme un oiseau. De même, l'invention du « monastère » fut précédée de celle d'une source, sveta-petkina voda. Les deux émergent sur un terrain familier, à proximité du moulin à eau possédé par son beau-père ${ }^{19}$ : un élément qui introduit le sujet de la propriété sur les sites révélés (question qu'il est délicat de poser). Comme je le constate par la suite, même avec un propriétaire bien identifié, un lieu saint n'est pas une «propriété » dans un seul, ni dans le seul, registre du social.

Le terme de monastère [manastir] appliqué à ce qui n'est qu'une petite chapelle ${ }^{20} \mathrm{~m}$ 'interpelle, ainsi que l'assertion qu'il était érigé « par les efforts de la famille [...] avec l'aide de tous les villageois » : ceci ne correspond ni à la terminologie, ni à la pratique de l'Église. De toute évidence, ici le mot a un sens spécifique de lieu sacré à la charge d'un groupe confessionnel territorialisé, et plus précisément d'une famille du village. S'apercevant de ma réaction face à l'emploi du terme manastir pour cette mini-église, la voyante précise : « l'église, c'est là où le village le veut - le monastère, c'est là où Dieu le veut » [crkva je deka selo saka, manastir je deka Gospod saka], avant de continuer son récit de vie mêlant l'histoire de plus d'une icône et celle du village. Pour l'ethnographe que j'étais à l'époque, la « découverte » d'un repère de sacralité chrétienne - une source (littéralement « une eau », voda) ou un sanctuaire - par une action visant à faire émerger du sol un objet préexistant, déjà là, était une idée insolite. De la même façon que la source sacrée était sur place, le sanctuaire dit « petit monastère » serait déjà là, attendant l'intervention de la visionnaire inspirée par « sa » sainte. Le déclenchement de cette révélation par voie onirique est similaire au rôle des rêves dans la découverte d'icônes étudié par Charles Stewart (2008). Ce déjà-làqu'il aborde par son aspect d'objet demandant à être révélé par une fouille (Ibid.,

19. Cf. note 14 supra. Le fait que les J. aient été (et soient toujours) connus comme des propriétaires de moulins à eau sis aux pieds du mont Belasica - évoqué par le fils de M. lors de mon dernier retour sur le terrain - apporte un éclairage supplémentaire, et autrement important, aux activités de la voyante.

20. C'est le diminutif - manastirče - qui est employé pour ce genre de constructions, à Bansko comme ailleurs dans la région de Strumica, et d'une manière générale en Macédoine du Nord. 
p. 98-99) - peut être interprété par la formule de l'« élaboration mentale » [imaginative mental processing] d'idées que Stewart relie à la propriété sur la terre, sur un territoire, à l'autochtonie : trois éléments enchevêtrés dans la perspective de la nation. Autrement dit, faire émerger de la terre, dans une localité partagée des siècles durant entre deux groupes confessionnels, une « eau bénite » [ayiasmo], a fortiori un « monastère », est la transposition symbolique des idées associées à l'État national et à l'ordre historique qu'il instaure.

Cette transposition s'opère dans le langage métaphorique de l'image. M. pense, pour ainsi dire, en icônes : que ce soit pour relater sa vie ou pour raconter l'histoire du village, elle met en scène des icônes de la Mère de Dieu et de quelques autres saints orthodoxes qui, selon ses récits, se seraient manifestés en chantant, en pleurant ou en volant ${ }^{21}$. Elle attribue des interventions miraculeuses aux icônes des saints emblématiques pour la communauté locale, en même temps établissant subrepticement une analogie entre le fonctionnement de ces objets-personnes et sa propre fonction de « porte ouverte » vers la sainte qui la possédait ${ }^{22}$. Une curieuse projection de sa relation mystique avec sainte Petka est sa photo d'identité accrochée dans un coin de l'icône de la sainte déposée dans l'église paroissiale. Plusieurs mois après le décès de la voyante, en 2000, la photo y demeurait, alimentant la croyance que la voyante recevait « là-haut » les honneurs adressés à l'icône de sa sainte.

Les récits de vie de $M$. et de plusieurs personnes de sa famille et de son entourage, les bribes de souvenirs et de récits concernant ses performances visionnaires dessinent une vision religieuse $\mathrm{du}$ monde largement partagée par les orthodoxes de cette Macédoine rurale au tournant des $\mathrm{XX}^{\mathrm{e}}$ et $\mathrm{XXI}^{\mathrm{e}}$ siècles, selon laquelle la femme (très rarement l'homme) « qui voit » - et non le prêtre dans l'église - est le médiateur entre la communauté sur terre et la sphère divine

21. La parole articulée est la seule manière dont la représentation de la Mère de Dieu ne se manifeste pas devant notre voyante - détail symptomatique, étant donné que cette dernière agit comme porte-parole de la sainte dont elle se revendique habitée ou « asservie ». La parole reste donc de l'apanage de l'agentivité humaine même quand celle-ci se donne comme subordonnée à la volonté divine.

22. La technique de communication de la voyante avec les saints est une variété de possession décrite comme un « asservissement » [zarobvane]. L'appellation locale, videne preko slava (vidi mi se preko slava), dénote la communication avec l'entité supranaturelle comme une révélation donnée par la vision mystique. En même temps, cette technique réfère explicitement au rituel de slava, la célébration annuelle du saint patron d'un patrilignage (cf. Rhewвоттом, 1975), détail invitant à traduire la possession comme « voir en communiant avec le saint ». 
chrétienne ordonnée autour de la Mère de Dieu et son Fils. Dans cette vision du monde, non seulement l'icône représente la figure sainte, mais elle est son corps un corps qui s'anime pour exprimer la volonté de l'invisible « prototype » qu'elle représente. En outre d'attribuer une corporéité et une intentionnalité à l'image de l'entité divine, cette vision s'exprime dans l'acte même de raconter, de relater une histoire d'icône miraculeuse - ou plutôt, la biographie de celle-ci, qui se trouve intimement liée à l'histoire locale et nationale. La question qui se pose est : peut-on généraliser cette vision du monde ? Et dans quelle mesure elle peut être toujours décelée?

\section{L’icône-qui-vole de Bansko/Konče : géographies légendaires et réelles}

Les récits retraçant la biographie de l'icône-qui-vole de Konče émergent successivement, produits dans des contextes sans cesse changeants. Ils dessinent différentes géographies du déplacement volontaire de cette icône que l'on va examiner à présent.

\section{« Sveta Bogorojca vivait à Bansko »}

Le premier récit, produit dans le contexte de la fête locale, émerge spontanément dans le récit de vie de la visionnaire, relié au fil narratif des révélations divines qui lui «ont été données ». Cette insertion-appropriation d'un motif légendaire à l'intérieur d'une expérience mystique personnelle, offerte à la communauté dans un acte narratif ritualisé, rend d'autant plus fort l'aspect biographique. Biographie personnelle et vie d'un objet s'interpénètrent et fusionnent, la biographie de l'icône croise celle de la voyante mais aussi celle de la collectivité ayant jadis habité ce village. La biographie de l'icône s'avère intimement liée à « l'ancienne église » de Bansko présentée comme l'habitat originel de la Mère de Dieu incarnée dans son icône - et à sa perte résultant de la rupture du lien unissant le groupe territorialisé avec sa patronne et protectrice céleste. C'est la disparition de la « maison » de l'icône qui aurait provoqué son action intentionnelle de quitter le lieu, entraînant la disparition de l'ancien village chrétien.

Autrefois, Bansko avait une grande église, dédiée à Sveta Bogorojca. Elle était plus bas, là où était l'ancien village. Une chrétienne décida de se marier avec un Turc, sans la bénédiction de ses parents... de changer son nom, de devenir turque [...]. Son père voulut la tuer... et Sveta Bogorojca se mit elle aussi en colère. Et lorsque cette femme passa près de l'église de la Vierge, celle-ci la paralysa, pour la punir de l'abandon de sa foi [chrétienne]. 
[...] Quand elle est devenue Turque, la femme décida de se venger... et jeta dans l'enceinte de l'église toute sorte d'impuretés, même des excréments. Ne pouvant endurer cela, Sveta Bogorojca voulut quitter le village et s'envola... Elle voulut aller bien loin, jusqu'à [la ville de] Kukuš [Kilkis en Grèce], mais dans son vol elle se heurta à un rocher là-haut dans la montagne [M. agite la main pour montrer] les Turcs d'ici l'appellent toujours ikona-kayası [turc : rocher de l'icône], et fut détournée de son vol. Elle se posa près de Lubnica, un village de chez nous... enfin, entre Lubnica et Konče [voir infra] là-bas, il y a de vielles églises grecques. Et lorsque les gens de ces deux villages trouvèrent l'icône, ils se la disputèrent... Finalement elle est restée à Lubnica. Et le village de Bansko, abandonné par sa Sveta Bogorojca, alla de mal en pis. Son église tomba en ruines, ses habitants moururent les uns après les autres. Celle qui fut à l'origine de tout cela attrapa une maladie incurable... Le village resta sans église, et bientôt il n'y eut plus de village.

Pour preuve de la véracité de son récit, la voyante évoque un repère tangible, des ruines. Effectivement, les ruines d'une église « très ancienne » furent trouvées dans les champs au nord du village où un chantier archéologique est installé de longue date ${ }^{23}$. Ce dernier est à proximité du lieu saint musulman le plus connu, le tekkélturbé d'Ismail baba, revendiqué de longue date par les orthodoxes comme « l'ancien monastère de Sveti Dimităr » [Démétrios de Salonique] ${ }^{24}$. L'histoire de l'icône volante était-elle inventée par la voyante, en une sorte de mise en histoire d'un motif légendaire destiné à inscrire les rapports entre les communautés du village dans une histoire longue, qui accorde l'antériorité, donc la priorité, aux chrétiens ?

23. Pour les fouilles de cet ancien lieu de culte chrétien, voir http://www.mpc.org.mk/ MPC/SE/bansko.asp (consulté le 11/02/2008), avec des réserves sur la chronologie. Quelques interlocuteurs de Bansko situèrent le début des fouilles archéologiques dans les années 1970, ce qui correspond à la période visionnaire très active de M.

24. Pour la confusion entre tekké et turbécf. CLAYER, 2010, p. 416. Revendication relevée déjà par Filipović, 1954, p. 12 ; il s'agit du même vestige du passé chrétien que la voyante a interprété comme «l'église ruinée de Sveta Bogorojca ». D'évidence on assiste ici à une confusion entre «église » et « monastère », cette dernière appellation étant préférée par mes interlocuteurs de Bansko. Dans ces échanges, trois saints chrétiens furent évoqués comme patrons antérieurs de ce lieu de culte musulman : saint-archange Michel, saint Dimităr, saint Charalambos. 
Les visites successives au village et les entretiens avec une pluralité d'interlocuteurs me font prendre la mesure de la popularité du récit entendu de $\mathrm{M}$. au sein de la communauté orthodoxe locale : la biographie de l'icône-qui-vole m'a été régulièrement racontée et certains détails, mis en discussion lors des réunions des orthodoxes de Bansko, ainsi que des groupes de croyants des villages proches et de Strumica venus commémorer la voyante après son décès. À ces occasions, l'histoire était reproduite par des proches de la visionnaire défunte, avec des altérations qui laissaient entrevoir l'effritement de son caractère normatif. Au fur et à mesure que les échanges autour de l'icône volante se multiplient, l'évocation du lien avec le village Bansko tend à disparaître tandis que le lien avec les villages de Konče et Lubnica dans le district de Radoviš, à une centaine de kilomètres au nord-ouest s'impose comme le noyau narratif stable.

\section{«Comment Sveta Bogorojca est allée à Lubnica »}

Quelques mois après le décès de la vidovita M., le récit de l'icône-qui-vole fut reproduit par sa belle-fille D., native de Lubnica ${ }^{25}$. Insistant sur la capacité de sa belle-mère de « voir » l'histoire de « ce village » et des « gens d'ici », D. reprend le récit à son compte, reproduisant fidèlement certains détails, s'écartant sensiblement d'autres. La version relatée par la belle-fille reprend l'histoire au moment où l'icône est retrouvée entre les deux villages voisins.

Les gens de Lubnica trouvèrent l'icône et l'apportèrent dans leur église, saints Constantin et Hélène. Mais ceux de Konče, le village où il y a une vieille église de la Mère de Dieu, décidèrent que c'était à eux, cette [icône de] Bogorodica, et s'en emparèrent. Mais elle s'est envolée et elle est retournée à Lubnica. Alors les habitants de Konče la fixèrent avec des clous et elle n'a pu bouger, et elle s'est résignée [à y rester]. Et depuis, on la porte chaque année à Lubnica, pour la fête de leur église - et elle s'en réjouit, et devient toute légère ainsi qu'une personne peut la porter allègrement ; alors qu'à son retour vers Konče, elle se met en colère, devient toute rouge et se fait si pesante qu'une seule personne ne peut jamais la bouger.

Cette version de la biographie de l'image volante place l'accent sur la querelle entre les deux villages pour posséder l'icône venue du ciel. La volonté de son

25. Entretien dans un cadre d'une commémoration familiale de la défunte en juillet 2000 ; âgée de 45 ans à l'époque, D. insistait sur l'implication de sa belle-mère à trouver une épouse « des nôtres » pour son fils et sur sa familiarité avec les affaires religieuses de son village natal. 
appropriation par chacune des deux communautés s'appuie sur deux principes différents : la localisation de l'objet précieux dans ou à la lisière du territoire villageois pour Lubnica, l'existence d'une vieille église - la « maison » d'une ancienne dévotion mariale, pour Konče. Le récit est exact sur le plan factuel : l'icône se trouve bien à Konče, dans l'ancienne église, que l'on peut traduire comme un triomphe du principe de l'ancienneté de la dévotion sur celui de la territorialité. Toutefois cela ne va pas sans résistances, et la querelle pour posséder la précieuse icône aboutit à un compromis : sa circulation ritualisée, une fois par an, entre les deux villages concernés. Cet élément scelle la proximité des deux villages au-delà de leur contiguïté spatiale, un lien que le récit effleure à peine : les chrétiens de Konče et de Lubnica ${ }^{26}$ ont formé un binôme endogame. Le port rituel de l'icône dans le village qui en est « privé » peut être lu comme un autre indice du lien spécial entre ces villages, à la manière de la circulation de la Mahoma pour la fête des Maures et chrétiens dans la région de Valence (Albert-Llorca \& Albert, 1995).

\section{L'icône à Konče}

Le fait se vérifie lors d'une brève visite de Konče en juillet 2002 : je découvre un gros village clairement partagé en deux quartiers confessionnels [mahalles], chrétien-orthodoxe et musulman. Mes interlocuteurs sont des Macédoniens orthodoxes ${ }^{27}$, tous des hommes, dont les deux gardiens de l'ancienne église où se trouve l'icône. La « vieille église grecque » du récit de $\mathrm{M}$. se révèle effectivement ancienne, construite dans les années 1360/1370 et faisant l'objet d'une mise en patrimoine pour ses fresques de la même époque. Posée à l'entrée de la nef, l'icône miraculeuse - peinte sur bois massif et très lourde - est effectivement fixée avec des clous sur une structure permettant de la soulever et la porter. Ce qui surprend, c'est le style de l'image et les signes matériels de la vénération qui lui est portée : la Vierge à l'Enfant qu'on aperçoit au milieu d'une exubérance de tissus et de plaques votives rappelle davantage les Vierges catholiques et tranche singulièrement sur le style byzantin des fresques ${ }^{28}$.

26. Konče est le chef-lieu de la municipalité du même nom comprenant sept autres villages, dont Lubnica. À la fin du XIX ${ }^{e}$ siècle les deux villages sont mixtes, avec une part substantielle de Turcs (KANČOv, 1996, p. 234-235) qui constituent toujours la majorité à Konče (dernier recensement de 2002).

27. Visite effectuée en compagnie d'un Macédonien de Bansko qui donna à cette plongée dans le terrain l'apparence d'une « visite de courtoisie », dont la durée ne devait pas dépasser la journée.

28. La question du style inhabituel de cette icône a préoccupé les historiens macédoniens de l'art religieux : je dois à M. Nikola Panzov, historien d'art du musée de Štip (2003), 
Sur place, seuls trois des cinq interlocuteurs déclarent connaître « la légende » de l'icône de leur église. Comparés aux narrations détaillées faites dans des contextes plus ou moins ritualisés par les femmes de l'entourage de M. à Bansko, les bribes de récits livrés par les hommes locaux se réduisent à quelques phrases concernant la « sortie de l'église » annuelle de l'icône. Il s'agit de l'occasion, un jour de fête précise (les avis divergent entre le jour des saints Constantin et Hélène ou de la Saint Sauveur), à laquelle l'icône est portée jusqu'à une localité limitrophe avec le village de Lubnica. Ce lieu n'est guère identifié comme celui de l'arrivée de l'icône « du ciel » : l'aspect de déplacement miraculeux est minoré, voire inexistant dans les récits des hommes de Konče. Ils se montrent bien plus préoccupés par le vivre-ensemble actuel dans leur village, insistant sur l'écart entre «nous » et les Turcs locaux. Ces derniers sont présentés comme étant riches et forts de leurs réseaux en Occident et avec la Turquie, et leur esprit entrepreneurial est opposé à l'apathie des Macédoniens/chrétiens locaux. Hormis les réflexions sur la division interne de Konče, seul le village voisin de Lubnica existe dans la narration sommaire de « la légende » de l'icône miraculeuse que j'y relève, où toute allusion à un conflit avec ce dernier est gommée.

\section{L'icône miraculeuse des folkloristes}

La question qui s'est posée après avoir relevé ces récits était celle des adaptations locales d'un motif itinérant à caractère historico-légendaire. Effectivement, une « légende de l'icône miraculeuse de Konče » est depuis longtemps consignée par les folkloristes ${ }^{29}$. Dans un tour d'horizon des matériaux publiés jusqu'aux années 1990, le folkloriste macédonien Tanas Vražinovski (1999, p. 125-126) fait le point sur les versions de « la légende de l'icône miraculeuse de Konče », sans chronologie ni mise en contexte des récits :

L'icône miraculeuse qui se trouve actuellement dans l'église Sveta-Bogorojca du village Konče, district de Radoviš, fut trouvée le jour des saints Constantin et Hélène dans la montagne au-dessus du

l'information que l'icône a été attribuée à un peintre d'icônes et photographe local, actif entre la fin du XIX ${ }^{\mathrm{e}}$ siècle et les années 1920. Il s'agirait de Kostadin Vangelov, formé en Voïvodine - région dans l'Empire des Habsbourg jusqu'en 1918 où les Serbes côtoient des Hongrois catholiques - pour qui on note un passage à Konče en 1918. Cf. BALABAnov, 1988, p. 42, 67-70.

29. VRAŽinovski, 1999, p. 125-128, avec des renvois aux folkloristes de la fin du $\mathrm{XIX}^{\mathrm{e}}$ siècle, dont Marko Cepenkov, considéré comme père fondateur de la science du folklore en République de Macédoine. 
village, et l'église y fut bâtie. Chaque année les villageois se rendent à l'endroit où l'icône fut trouvée, lors de cette fête ; ils y font bénir de l'eau pour la santé et la bonne récolte que Sveta Bogorojca est censée assurer. Le jour du saint Sauveur [l'Ascension, dans le calendrier orthodoxe] les gens se réunissent dans l'église et portent l'icône à l'extérieur. L'icône doit être portée par un membre de la famille qui l'avait trouvée : il la porte jusqu'à l'endroit où elle fut trouvée, et l'y dépose. Alors l'icône commence à suer [da se poti], un signe qu'elle est miraculeuse. Par peur des Turcs, certains ont décidé de ne plus sortir l'icône de l'église. Cela a duré quelques années : pendant ce temps les gens et le bétail avaient des problèmes de santé, et les champs ne donnèrent pas de bonne récolte. Pour cette raison ils ont décidé de retourner à la coutume de sortir l'icône de l'église, ce même jour.

Une fois de plus, le cadre territorial d'action de l'icône miraculeuse change : le village voisin disparaît complètement de ces versions, ainsi que toute idée d'une circulation de l'icône entre deux villages voisins. Toute l'activité rituelle est expliquée par rapport à une borne calendaire (la fête des saints Constantin et Hélène) et spatiale de « découverte » de l'icône (sans rapport à un autre village). Le contexte ottoman apparaît plus nettement dans ce récit générique, pour ainsi dire, ce qui correspond bien à la tendance (implicite ou explicite) chez les folkloristes à considérer ces récits comme un écho des « réalités historiques 》. L'élément moralisateur est appuyé : l'interruption de la procession avec l'icône « par peur des Turcs » conduisant à des malheurs révélateurs de la volonté de Bogorojca, la procession annuelle est reprise, avec l'effet bénéfique attendu.

Toutefois l'agentivité de l'objet-personne semble minorée : la Mère de Dieu n'exprime plus sa volonté en s'envolant, ni en changeant de poids (devenant légère quand elle approuve un déplacement, ou très lourde quand elle désapprouve), mais en dégageant du liquide, assimilé à l'acte de «suer ». Ce terme, désignant une fonction physiologique d'un corps vivant, dégageant des humeurs, est ici le seul signe de la capacité d'agir de l'icône miraculeuse qui est ainsi traduite dans un registre du sensible. Des icônes qui suintent sont bien connues dans les contextes balkaniques et au-delà, chez les chrétiens du Proche Orient (Syrie, Liban), et l'écoulement d'un liquide de l'image peut également être perçu comme des larmes (Aubin-Boltanski, 2012a, p. 102 et suiv.). Qu'il s'agisse de «larmes » ou de « sueur », l'assimilation de l'image au corps humain transpose la capacité d'agir de l'icône dans le domaine du sensible et l'inscrit dans un champ sémantique associé à l'effort ou à la souffrance. 


\section{Biographie de l'icône selon le rapport d'un évêque orthodoxe bulgare}

À la lumière de ces données on peut se demander si la mise en relation de la célèbre icône-qui-vole avec le village de Bansko n'est pas le seul fait de l'inventivité de la voyante. Il s'avère cependant que ce lien est documenté au début du $\mathrm{XX}^{\mathrm{e}}$ siècle, dans le compte-rendu que le dernier métropolite bulgare de Strumica, Gerasim, a fait des lieux de culte orthodoxe de son ancien diocèse ${ }^{30}$. Dans son Rapport concernant l'état des églises et des monastères dans les parties libérées du diocèse de Strumica ${ }^{31}$, ce dernier accorde une attention particulière au « monastère Sveta Bogorodica près du village de Konče » qu'il affirme avoir visité à quelques reprises « à l'époque turque » (avant 1912). Ne disposant d'aucune indication vérifiable sur la date de sa fondation, il s'appuie sur « la tradition orale » collectée dans les villages autour de cette ville pour étayer l'hypothèse de sa fondation après l'établissement des « Turcs » (Ottomans) dans la région.

Selon la tradition [predanieto], à Bansko, près de Strumica, il y avait un grand monastère dédié à saint Charalambos qui était connu dans toute la région, car Bansko était le plus grand village du district de Strumica. Ici, jusqu' il y a 200 ans, ou plus, se tenaient des « foires de lin » [keten panayir], au nombre de six [par an], où venaient commercer des marchands d'aussi loin que Vienne. Ce commerce rendait le monastère florissant, il avait de gros revenus. Les Turcs l'ont détruit et l'ont transformé en tekké de leurs sectaires [illisible]. À l'époque turque ici, des Turcs et des chrétiens allumaient des cierges sur le tombeau [grob] de saint Charalambos. Le monastère ruiné, deux de ses icônes se sont retrouvées ailleurs l'une à Konče, l'autre dans les Poroya ${ }^{32}$. Là où ces icônes furent

30. La métropolie de l'Exarchat bulgare a été établie à Strumica en 1897 ; trois métropolites s'y sont succédés avant que le diocèse ne passe en territoire serbe. Le fonctionnement de ce siège est très perturbé déjà pendant les guerres balkaniques de 1912-1913 (Strumica est prise par les Serbes au printemps de 1913) et surtout pendant la Première Guerre mondiale, au terme de laquelle la ville est attribué au royaume des Serbes, Croates et Slovènes (1920). La métropolie bulgare de Strumica est alors supprimée, le métropolite étant contraint à se retirer dans le royaume de Bulgarie, où il réside déjà pendant la Grande guerre et d'où il produit le rapport.

31. Préservé dans les Archives d'État de Blagoevgrad [Bulgarie], Fond 48K, op. 1, unité arch. 29.

32. Il s'agit des villages actuels d'Ano Poroya et Kato Poroya en Grèce, sur le versant sud du mont Belasica. 
trouvées, des monastères furent érigés : ainsi fut fondé le monastère du village de Konče dont il est question.

La version rapportée par le métropolite Gerasim confirme la primauté de Bansko dans la biographie de l'icône miraculeuse. La trame du récit de M. se retrouve donc dans un récit de toute autre nature, le rapport [relatio] du haut prélat d'une Église orthodoxe politisée et prisonnière de l'idéologie nationale, qui reconstitue le passé des grandes fondations de son ancien diocèse en se tournant vers les « on-dit historiques ${ }^{33}$ ». Konče, le village qui possède l'icône de nos jours, y est présenté en position de bénéficiaire après la ruine de « l'ancien monastère » de Bansko tout comme l'autre village situé sur le versant opposé de la montagne.

Ce dernier récit est aussi le seul à mentionner explicitement l'installation d'un foyer d'islam hétérodoxe dans ce lieu de culte chrétien. La ruine du haut lieu chrétien est attribuée par notre évêque à une politique intentionnelle des «Turcs » (Ottomans), ce qui n'est pas étonnant dans le contexte de production du rapport, en pleine Grande Guerre. Bien attestée dans le contexte ottoman, la conversion d'églises en mosquées est un « fait historique » exploité par les idéologies nationales des États du Sud-Est européen et présenté comme la preuve de la violence structurelle anti-chrétienne de l'Empire ottoman. Ici, le fait semble corroboré par les observations d'un ethnographe serbe (Filipović, 1954, p. 12-13) qui relève la vitalité de l'ordre des Bektachis ${ }^{34}$ à Bansko et dans quelques villages voisins ainsi que la vogue de la croyance relative à l'origine chrétienne de leur monastère. Le plus intéressant cependant est qu'à côté de ce qu'il semble être une rivalité interconfessionnelle, le prélat orthodoxe rapporte l'existence simultanée du phénomène que la recherche actuelle qualifie de dévotion mixte (Albera \& Couroucli, 2009 ; Barkan \& Barkey, 2014) : des croyants des deux communautés religieuses brûlent des cierges au « tombeau »du saint chrétien. Trifunoski (1979, p. 100) y relève la même pratique en 1974, ainsi que celles de nouer des fils et accrocher du linge au fenêtres de la tekija [tekké] du saint bektachi Ismail baba. À la lumière du premier et du dernier récit, l'« ancien monastère [orthodoxe] de

33. C'est la traduction la plus appropriée du terme predanie qui signifie littéralement «ce qui se transmet [predava] par la bouche » mais dont l'emploi est réservé à des faits publics et des événements historiques.

34. Confrérie musulmane (islam hétérodoxe) très influente à l'époque ottomane dans les Balkans : voir Popović, Veinstein, 1994, et Clayer, Popović, 1992, pour l'aire qui nous intéresse. 
Bansko » transformé en tekké se révèle être un lieu de culte partagé entre chrétiens et musulmans hétérodoxes, très similaire à la dévotion au saint Nikola chrétien et au saint bektachi Hadır baba observée ailleurs en République de Macédoine ${ }^{35}$.

Ainsi au fur et à mesure de l'enquête, la biographie de l'icône se précise par une agentivité sur le plan historique en écho à celle des humains. L'icône-qui-vole apparaît comme opérateur symbolique de la vie des communautés humaines dans la longue durée et en particulier dans des moments cruciaux de leur existence, transposant à l'échelle locale des processus de vivre-ensemble et de division, de conflits et de partages.

\section{S'envoler pour fuir ? Trajectoires de l'icône volante de Bansko}

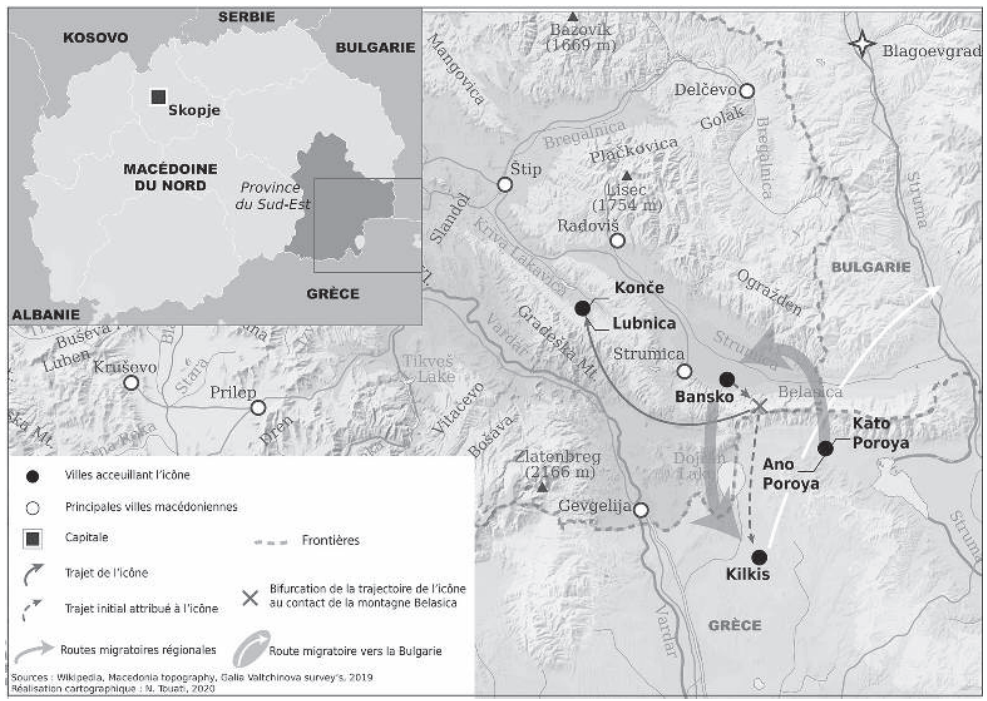

Les versions de la légende de l'icône-qui-vole semblent dessiner une sorte de géographie dévotionnelle à laquelle participent plusieurs villages et une ville. Mais s'agit-il uniquement, ou surtout, de géographie dévotionnelle ? Peut-on la ramener à d'autres registres du social ?

Bansko et Konče sont des villages éloignés et appartenant à deux districts différents (cf. carte), mais à y regarder de plus près, il y a entre eux une forte

35. Sur ce cas, qui a fait le miel des anthropologues, voir Bowman, 2010, p. 200-206. Une ethnographie filmique de cette dévotion est réalisée par l'ethnologue macédonienne Elisabeta Koneska, «Lieux saints partagés »/“Peace for all”, https://www.youtube.com/ watch?v=QJbK_y36nZM (visité le 17/09/2019). 
similarité. De gros villages mixtes à forte majorité musulmane (les groupes confessionnels qui y cohabitent s'identifient dans les deux cas comme Turcs et des Macédoniens), ils sont tous les deux liés à d'anciennes fondations religieuses connues bien au-delà de l'échelle locale, et présentent un profil démographique et un paysage religieux similaire. Au moment de l'enquête, le lien entre les deux villages est établi uniquement par le récit de la voyante et l'acte narratif est central dans l'entretien de ce que l'on peut qualifier d'une mémoire de proximité de destin - mémoire qu'elle a réactualisée par ses rêves-visions. Par ailleurs, Bansko est entouré de villages à forte identité grecque ${ }^{36}$, fait que la voyante a bien connu pour être originaire de l'un d'entre eux (Robovo). Consciente de son propre rôle de gardienne de la mémoire collective, ses récits mêlent l'histoire de sa vie, de son village natal et celui de sa résidence matrimoniale, de sa communauté, à la biographie de l'icône volante.

La narration établit un lien entre deux autres villages, Konče et Lubnica, lien qui est bien tangible dans les réalités : villages voisins dont les territoires sont contigus, ils ont formé pendant des siècles une communauté endogame pour les chrétiens. Un autre groupe est formé par les villages appelés « les Poroya » (Kato et Ano - gr., d'en bas et d'en haut) vers lesquels est censée aller l'autre icône restée orpheline après la ruine du monastère de Bansko (selon l'information rapportée uniquement par le métropolite Gerasim). Ces deux villages de grande taille, sis sur le versant sud du mont Belasica dont la crête marque, depuis 1913, la frontière entre la Grèce et le pays sud-slave au Nord (la « Serbie du Sud », puis la République de Macédoine), se caractérisent eux aussi par la mixité. Marqués comme «bulgares » dans la dernière décennie du $\mathrm{XIX}^{\mathrm{e}}$ et au début du $\mathrm{XX}^{\mathrm{e}}$ siècle en raison de l'adhésion de leur population à l'Exarchat bulgare, à la fin de l'époque ottomane (1912) ils présentent un conglomérat ethnique entre Bulgares, Valaques et Turcs (Kančov 1996 [1900], p. 185) où l'identification grecque au sein des deux premiers groupes va de pair avec l'adhésion au Patriarcat de Constantinople. Enfin, il y a la ville de Kukuš/Kilkis, mentionnée comme destination première de l'icône volante dans le premier récit, qui est située à une centaine de kilomètres au sud de Bansko, en Grèce.

Que relie tous ces lieux (cf. carte) au-delà des différentes trajectoires prêtées à l'icône-qui-vole ? Une fois de plus, on trouve la clé pour comprendre cette géographie hautement symbolique dans les récits de $\mathrm{M}$. La plupart de ces trajectoires correspondent à des mouvements migratoires des populations de la

36. TRIfunoski, 1979, p. 101, parle de la force du « parti grec »d'avant 1913 dans plusieurs villages voisins dont Mokrievo, Kolesino, Veljusa. 
région de Strumica au moment de la « nationalisation » de la société locale - un long moment qui s'étend sur plusieurs décennies, entre la fin du XIX ${ }^{\mathrm{e}}$ siècle et l'après Seconde Guerre mondiale. Ces mouvements accompagnant le démantèlement de l'Empire ottoman et la mise en place des États modernes dans cette partie des Balkans, désignés à l'époque par le terme d'《échange de populations » - que l'on tend a posteriori à décrire en termes de migration forcée ${ }^{37}$ - ont leur apogée dans la décennie 1912-1922. Ainsi la ville de Kilkis est le lieu de refuge pour les habitants locaux de culture et d'identité grecque en 1913 : la volonté de l'icône de «s'envoler vers Kukuš » correspond précisément au déplacement massif de la population de Strumica et des villages au sud-est de la ville qui se reconnaît dans le projet hellénique moderne. Comme cela a été démontré (Embirikos, 2011), la conquête de cette région par le royaume de Grèce en 1913 fut accompagnée de violences massives. La violence est présente de tous les côtés : convoitée de trois partis belligérants (grec, bulgare et serbe) dans les guerres balkaniques de 1912-1913, l'absorption de cette région par un État national eut pour résultat le déplacement massif de populations du nord au sud (pour les hellénophones) et du sud au nord (pour les « slavophones »). La plus grande partie des populations hellénisantes du district de Strumica, restée en dehors de la Grèce, part donc vers Kukuš/Kilkis, où des associations de « réfugiés » [prosfyies], dans le langage de l'époque, ou d'exilés, continuent à cultiver une mémoire de perte et de deuil ${ }^{38}$. Dans les années 1920, l'aire de Kilkis vit l'installation massive de « réfugiés » grecs d'Asie Mineure à qui fut confié le rôle de « gardiens de l'État-nation » (Bruneau 2015).

37. Depuis la dernière décennie $\mathrm{du} \mathrm{XIX}^{\mathrm{e}}$ siècle et jusqu'aux échanges de populations pratiqués avec une sanction internationale après la Première Guerre mondiale, plusieurs vagues de déplacement sont attestées amenant des populations grécophones des villages de la plaine de Strumica vers la Grèce : voir Trifunoski, 1979, p. 13-17 et passim. Rarement volontaires, ces déplacements opérés dans une logique de « réunification avec la Mère Patrie » furent presque toujours consentis, ce qui n’empêche que les déplacés et leurs descendants parlent d'exil.

38. Lors d'un bref sondage de terrain effectué à Kilkis en 2000, j'ai pu observer des attitudes de recueillement devant deux panneaux commémoratifs posés par des associations d'exilés dans l'église des Saints Quinze martyrs de Tiverioupolis : excentrée mais bien visible du centre-ville de Kilkis, cette église de construction récente est dédiée aux saints patrons de l'église principale et la plus ancienne de la ville de Strumica. Au-delà des saints-patrons, le lien avec Strumica et sa région se lit dans la multitude d'inscriptions de donateurs (pour la fabrication de la décoration intérieure de l'église) provenant de cette dernière ville et des villages de ses alentours. 
L'intention lors de l'envol de l'icône traduit la volonté propre [ona sakala] de Bogorojca/ la Mère de Dieu d'aller vers la Grèce : l'icône, imaginée comme une incarnation de la Vierge, possède la matérialité corporelle qui en fait la métonymie d'un corps collectif. Cette volonté de fuite et d'installation à Kukuš/ Kilkis peut se traduire de deux façons : lieu propre au sens de « non souillé » dans un sens religieux, où l'accent est mis sur l'absence de mixité - mais aussi, lieu propre ${ }^{39}$ car identifié à un État-nation, à une nation supposée homogène d'où l'Autre religieux et les ethniquement autres se sont retirés ou en furent chassés. Et si l'icône «veut rejoindre » Kukuš/Kilkis, il s'agit d'une manière d'exprimer le fait que c'est précisément vers cette ville que convergent des populations à identité grecque de la plaine de Strumica, y compris du village natal de M., sensible aux mouvements migratoires aussi bien de par son propre vécu que par l'expérience de sa belle-famille venue d'Ano Poroya.

Cette volonté ne reste pourtant qu'une intention : le vol de la Mère de Dieu est entravé, de même que la capacité d'agir des collectivités humaines qu'elle accompagne et protège se trouve contrariée par une capacité d'agir humaine à une échelle supérieure, celle de l'État national ${ }^{40}$. Ainsi Sveta Bogorojca de Bansko atterrit à l'opposé de sa destination initiale. Son intention est cependant respectée : quitter sa « maison » et $s a$ communauté souillée et aller rejoindre une chrétienté plus ancienne et supposée « pure ».

\section{Langage symbolique et discours identitaire de l'icône-qui-vole}

Si l'icône de la Mère de Dieu «s'envole », c'est pour s'enfuir d'un lieu pollué au sens propre comme au sens figuré, illustrant le lien établi par Mary Douglas (2005) entre propreté et sacré. Identifié à « l'ancienne église » dans le récit de la voyante, ce lieu est identique à « l'ancien monastère » dans la tradition transmise par le prélat orthodoxe. Dans les deux versions, l'icône s'enfuit car le lieu est pollué ou approprié (ce qui revient au même) par l'autre religieux. À la version (rationalisée ?) de la conversion du lieu de culte s'oppose celle de la conversion d'un être humain acteur de son destin. Car dans le récit de la voyante, la Mère de

39. Ici, « lieu propre » est pris dans le sens que lui donne CERTEAU, 1990 [1980], p 31-34, dans sa proposition de distinguer entre tactiques et stratégies : avoir « un propre », ou un lieu propre, connote la capacité d'agir.

40. Voir Embirikos, 2011 pour de nombreux exemples d'empêchement de déplacements souhaités ou bien d'encouragement de départs non souhaités, lors de l'échanges de populations entre la Grèce et la Bulgarie, de la part des deux gouvernements. 
Dieu «s'envole » en réponse à une autre agentivité féminine présentée comme malfaisante : la démarche d'une chrétienne convertie à l' islam de sa propre volonté. Il n'est pas un hasard que cette dernière narration soit produite dans un village mixte, où la question de ce type d'intermariage a dû se poser à maintes reprises.

Dans le cadre ottoman, dont le souvenir est toujours vif et souvent évoqué dans la société locale de Bansko au début du XXI ${ }^{\mathrm{e}}$ siècle, les unions entre chrétien(ne)s et musulman(e)s furent généralement admis dans un sens, pour un musulman prenant femme en dehors de sa communauté. La femme prise demeure sous pression de conversion, laquelle se réalise tôt ou tard (Sant-Cassia, 2018, p. 127). Le mariage interconfessionnel étant craint comme un élément susceptible de détruire l'intégrité du groupe « donneur de femmes » et mettre en danger sa survie, un discours anti-conversion adressé au femmes chrétiennes est palpable assez tôt (Krstić, 2011, p. 157-161). Il est d'autant plus fort au XIX ${ }^{\mathrm{e}}$ siècle, le siècle des réveils nationaux, lorsque les premières collectes de folklore mettent au jour le motif du « rapt » de femmes chrétiennes et de leur conversion forcée pour être mariées à des musulmans. Ces matériaux de littérature orale deviennent rapidement la cible de manipulations et de recherche de correspondances entre « la mémoire du peuple » et une vérité historique. De fait, le folklore met des paroles et traduit en motifs (racontés ou chantés) une norme que l'Église orthodoxe s'efforce d'imposer de longue date, et qui ne laisse aucune place à une possibilité d'unions volontaires, par amour - une réalité pourtant palpable dans les sources historiques. Même dans les cas où la conversion est consentie ou souhaitée par la femme, la rumeur publique concernant de telles unions (ou leur préparation) a pu conduire à des réactions violentes allant jusqu'à des heurts intercommunautaires ${ }^{41}$.

Se marier dans un ailleurs confessionnel, pour la femme, signifie la mise en danger du groupe qu'elle quitte : un tel mariage ouvrirait une brèche par laquelle la communauté risque de perdre son «sang ». Il y a plus de trente ans, l'ethnolinguiste Georges Drettas (1985) a proposé une lecture de l'imaginaire du vampire dans les Balkans comme le « sang perdu » de la communauté chrétienne qui perd ses femmes. Venant du Nord de la Grèce, d'une région proche du lieu d'ancrage de cette étude, cette réflexion présente un parallèle saisissant avec ce que la voyante exprime par le langage métaphorique de l'icône-qui-vole.

41. Deux cas provenant de deux villes multiculturelles, Salonique en 1876 et Roussé (sur le bas Danube) en 1910, sont examinés dans Gradeva \& Kuneralp, (in press), qui montrent que l'affectivité n'est pas prise en compte : ce qui compte, c'est le respect d'une norme perçue comme assurant la survie communautaire. 
Si on relit le récit de la voyante de Bansko par ce prisme, l'optique identitaire apparaît encore plus clairement. Ouvrant la voie à l'une des érosions les plus dangereuses pour la communauté, l'union volontaire de la femme chrétienne avec un « Turc » est sanctionnée par le châtiment divin. Celui-ci est porté par la Mère de Dieu ou par son hypostase matérielle qui habite le sanctuaire le plus proche : Sveta Bogorojca se trouve non seulement dans un rapport de proximité avec «sa » communauté chrétienne (Herzfeld, 1990, p. 112), elle incarne l'autorité de la communauté et celle du groupe territorialisé sur les femmes ${ }^{42}$. De manière inattendue - ou plutôt, qui n'a pas lieu d'être - la réaction de la femme convertie est de « se venger », punir à son tour la Mère de Dieu en polluant sa demeure, lieu saint pour les chrétiens orthodoxes. L'ultime résultat de cet enchaînement de punitions est la fuite de la Mère de Dieu sous l'aspect d'envol de son icône. La pollution déclenche une mutation identitaire - la perte de l'identité chrétienne qui va jusqu'à la destruction physique de la communauté et sa disparition.

La relation entre « une femme » chrétienne et Bogorojca est celle de la survie du groupe - d'un groupe imaginé au-delà des liens du sang, comme étant soudé par la religion. C'est la relation de l'identité collective qui repose sur le sang et la reproduction biologique, mais aussi sur la « reproduction »du corps social soudé par la foi. Les deux registres se retrouvent dans l'imaginaire de la nation comme un corps. Comme Michael Herzfeld (1997, p. 74-88) l'a bien démontré, l'imaginaire de la nation comme un corps, enraciné à l'aide d'usage de métaphores corporelles, est d'une efficacité redoutable précisément parce qu'elle parle au plus profond de l'humain et passe par des catégories tangibles au quotidien. L'icône volante de nos récits est le corps de la Vierge/Mère de Dieu, figure divine des chrétiens dont la capacité à exprimer l'identité collective à une pluralité d'échelles n'a plus à être prouvée. Qu'il soit pris à l'échelle de la famille, du village, de la ville ou de la nation, le corps social est symboliquement représenté par la Mère de Dieu qui se manifeste dans et à travers son icône. Cette matérialité ouvre une capacité d'agir supplémentaire à la Vierge « sujet de l'Histoire ».

42. Dans les sociétés balkaniques orthodoxes, l'autorité sur les jeunes filles est traditionnellement détenue par leur père, fait auquel le motif de la punition paternelle voulue dans le premier récit est bien conforme. Dans les affaires de conversion à l'islam de jeunes filles chrétiennes étudiées par Gradeva \& Kuneralp, (in press), ce sont cependant les mères qui assument le rôle plus actif. 


\section{L'icône, la mémoire, l'histoire}

Pour conclure, un constat s'impose : une icône miraculeuse est à la fois une entité et un opérateur symbolique ; dotée d'agentivité, elle fait corps avec la société qu'elle est censée incarner et protéger. Sa biographie est celle du corps social - sa mémoire, son histoire - par-delà la condition structurelle de croire au miracle, au-delà même de la communauté croyante.

Saisie dans un premier acte narratif et suivie à travers quelques autres récits, la biographie de l'icône volante de Bansko/Konče s'articule autour de crises (au sens premier de terme grec krisis) - des moments critiques ou des bifurcations dans l'existence des communautés qui s'identifient à elle. Cette biographie se déploie autour de deux axes : celui de la séparation d'une communauté qui n'est plus la « sienne », et celui de l'accueil/intégration à une communauté qui l'adopte et se voue à elle. C'est le pôle de la disjonction et la perte qui est au cœur des récits de la voyante de Bansko, et cela de deux manières : comme disparition de la communauté d'ancrage par sa dissolution dans l'autre religieux - ou comme sa disparition par un mouvement de fuite ailleurs dans une perspective d'unité ethno-territoriale. L'un et l'autre constituent des interfaces entre ordres historiques différents, dans le vocabulaire de Stewart (2012), qui articulent le passage d'un ordre impérial (l'Empire ottoman) à un ordre stato-national.

Étant donné la densité du contexte de narration à Bansko, ce serait une erreur méthodologique de séparer les récits de l'icône-qui-vole des récits d'invention de lieux saints chrétiens. Comme on l'a déjà observé, l'invention de sources d' « eau bénite » et de « petits monastères » est pensée comme indépendante de la volonté humaine, déclenchée par intervention divine survenant au terme d'un processus de façonnage de la figure de médiation humaine, la visionnaire mettant en œuvre cette volonté. Au cœur de ce façonnage, c'est la capacité de la femme-qui-voit (et qui dit) à historiciser le rapport à l'objet ou l'objectif sacré qu'elle devait littéralement faire sortir de la terre, à l'installer dans une temporalité d'avant un événement traumatique lors duquel il fut « caché » ou « oublié ». Sans surprise, l'événement qui fait frontière est l'établissement de l'islam dominant, alias « les Turcs » ou l'Empire ottoman. L'icône volante, tout comme les icônes cachées et révélées dans les contextes insulaires grecs du XIX $^{\mathrm{e}}$ siècle (Stewart 2008), marque un changement tout à la fois dans le régime foncier (usage et dévolution de la terre mais aussi de l'accès à l'eau) et des droits « historiques » de possession d'un territoire. Ces deux modalités de manifestations d'une icône miraculeuse sont des marqueurs par excellence d'une communauté chrétienne préexistante à l'islam, 
la révélation et l'affirmation même de cette antériorité par des récits devenant l'emblème de la chrétienté menacée ${ }^{43}$.

L'acte narratif est central dans la biographie de l'icône volante et, plus généralement, dans l'entretien de ce que l'on peut qualifier d'une mémoire historique sans cesse réactualisée par des rêves-visions. Quid alors de la biographie de l'icône-qui-vole de Bansko/Konče une fois la gardienne de cette mémoire disparue ? Trois ans après la mort de M., un monastère «vrai, avec des moines » (pour reprendre la formule d'une orthodoxe du village), est érigé à Bansko à l'instigation de la hiérarchie supérieure de l'Église orthodoxe du pays. Visible de loin, le bâtiment principal est sis entre l'église paroissiale et le cimetière, effaçant les captages de sources d'eau bénite [sveti vodi] découvertes par la visionnaire. La nouvelle fondation est placée sous le patronage des Saints-Quarante Martyrs de Sébastée par référence à une « ancienne église épiscopale » présumée avoir existé aux abords de Bansko, qui n'est autre que «la vielle église de Bogorojca » dans la narration de la voyante ${ }^{44}$. Désormais dans l'enceinte du nouveau monastère, le manastirče Sveta-Petka est dépouillé de l'inscription mémorielle et de ses nombreuses icônes, avant d'être reconstruit comme petite église peinte selon les canons pour les services des moines. La petite communauté monastique appartient au courant néo-hésychaste pratiqué dans certains monastères du Mont Athos et observe un silence strict, ce qui rend difficile tout échange ${ }^{45}$. Il n'est plus question de relater l'histoire de l'icône volante de Bansko, ni celles des « eaux bénites » et autres lieux sacrés que la vidovita žena a «fait sortir de la terre ». Depuis la mainmise de l'Église orthodoxe sur le lieu saint révélé à Bansko, tout un pan de la biographie de l'icône volante semble suspendu. Cependant la construction de la même chaîne causale reliant la naissance du nouveau monastère aux ruines d'une

43. Aubin-Boltanski, 2012a, p. 113, observe (par rapport à Notre-Dame de Kazan) le même enchaînement entre une visionnaire « découvrant » l'objet « caché », les miracles, la consécration et la révérence des puissants, avant la transformation de l'icône en «bannière » d'un territoire politique et en emblème de la chrétienté menacée.

44. Voir http://www.mpc.org.mk/MPC/SE/bansko.asp (consulté le 21/06/2009) : le discours insiste sur la continuité entre la nouvelle fondation et l'ancienne « église épiscopale Saint-Quarante Martyrs » dont la substruction, datée « de l'époque des Comnènes », serait trouvée lors de fouilles archéologiques tout près de cet endroit.

45. Cf. https://www.youtube.com/watch?v=K8ajIIb0GOc (consulté le 10/08/2017). Un seul moine reçoit les gens de l'extérieur et répond à un nombre limité de questions. Cette orientation fut explicitement mentionnée par le moine préposé à la communication avec qui j'ai pu m'entretenir en septembre 2007. 
LES « BIOGRAPHIES » D'UNE ICÔNE VOLANTE ET LE STATUT DE QUELQUES RÉCitS DANS UNE RÉGION BALKANIQUE

« très ancienne » fondation à l'autre bout du village, atteste qu'un mécanisme similaire est toujours à l'œuvre. À quand le récit de l'icône volante sur Internet ?

\section{Bibliographie}

Albera Dionigi \& Couroucli Maria (dir.), 2009, Religions traversées : lieux saints partagés entre chrétiens, musulmans et juifs en Méditerranée, Actes Sud, Paris/Aix-en-Provence, 359 p.

Albert-Llorca Marlène \& Albert Jean-Pierre, 1995, « Mahomet, la Vierge et la frontière » in Annales $H S S$, n $^{\circ} 4$, p. 855-886.

Allard Olivier, 2010, «Objets, personnes, esprits » in Gradhiva, $\mathrm{n}^{\circ} 11$, p. 207-213, https://doi.org/10.4000/gradhiva.1767.

Appadurai Arjun (ed.), 1986, The Social Life of Things. Commodities in Cultural Perspective, Cambridge University Press, Cambridge, 329 p.

Aubin-Boltanski Emma, 2012a, « La très petite image de Notre-Dame de Soufanieh » in Archives des Sciences sociales des Religions, n 174, p. 101-112.

Aubin-Boltanski Emma, 2012b, « Notre-Dame de Béchouate : un “objet-personne" au cœur d'un dispositif cultuel » in L'Homme, no 203/204, p. 291-320.

Balabanov Kosta, 1988, La Galerie d'icônes, $2^{\mathrm{e}}$ éd., Institut de conservation des monuments de la culture de Stip, Stip, 123 p.

BARKAN Elazar \& BARKey Karen (eds.), 2014, Choreographies of Shared Sacred Sites, Columbia University Press, New York, 440 p.

Bloch, Maurice, 1995, « Mémoire autobiographique et mémoire historique du passé éloigné » in Enquête, n 2, p. 59-79.

Bowman, Glenn, 2010, “Orthodox-Muslim Interactions at 'Mixed Shrines' in Macedonia" in Hann C \& Goltz H. (eds.), Eastern Christians in Anthropological Perspective, University of California Press, Berkeley/Los Angeles, pp. 195-219. 


\section{CAHIERS DE LITTÉRATURE ORALE}

50 Éclats de paroles $-n^{\circ} 85$

BRU Josiane, 1999, « Le repérage et la typologie des contes populaires. Pourquoi ? Comment ? » in Bulletin de l'AFAS, n 14, https://doi.org/10.4000/afas.319.

Bruneau Michel, 2015, «L'installation des réfugiés grecs pontiques au Nord de la Grèce (Florina, Kilkis, Serrès) : leur rôle de gardiens des frontières de l'État-nation (1920-2000) » in Études Balkaniques. Cahiers Pierre Belon, n 21 , p. 93-116.

Christiansen Reignar Th., 1958, The Migratory Legends, a Proposed List of Types with a Systematic Catalog of the Norvegian Variants, Academia Scientiarum fennica (coll. FF. Communications, n 175), Helsinki, 221 p.

Clayer Nathalie, 2010, “Tekke” in Encyclopaedia of Islam, Tome X, New Edition, Brill, Leiden, pp. 416-425.

Clayer Nathalie \& Popović Alexandre, 1992, « Sur les traces des derviches de Macédoine yougoslave » in Anatolia Moderna/Yeni Anadolu, IV,Derviches des Balkans, disparitions et renaissances, Librairie d'Amérique et d'Orient/Institut français d'Études anatoliennes, Paris/Istanbul, p. 13-63.

Clerc-Renaud Agnès \& Leguy Cécile (dir.), 2016, Des vies extraordinaires : les territoires du récit, Cahiers de littérature orale, $\mathrm{n}^{\circ} 79$, https://doi.org/10.4000/ clo. 2538 .

Couroucli Maria, 1995, «Le lalein et le graphein, parler et écrire en grec » in Revue des Mondes musulmans et de la Méditerranée, $\mathrm{n}^{\circ}$ 75-76, p. 257-271.

Certeau Michel (de), 1990 [1980], L'Invention du quotidien, I. Arts de faire, Gallimard, Paris, 352 p.

Detelić Mirjana, 2010, "Saint Paraskeve in the Balkan Context" in Folklore, 121(1), pp. 94-105. DOI: 10.1080/00155870903482049.

Douglas Mary, 2005 [1966], Purity and Danger: An Analysis of Concepts of Pollution and Taboo, Routledge and Kegan Paul, London, 179 p.

Drettas Georges, 1985, «Questions de vampirisme » in Études rurales, no $97-98$, p. 201-218. 
LES « BIOGRAPHIES » D'UNE ICÔNE VOLANTE ET LE STATUT DE QUELQUES RÉCITS DANS UNE RÉGION BALKANIQUE

Embiricos Leonidas, 2011, « Kilkis 1913 : territoire, population et violence en Macédoine » in European Journal of Turkish Studies, ${ }^{\circ}$ 12, http://ejts.revues. org/4486 (consulté le 30 septembre 2016).

Filipović Milenko, 1954, "The Bektashi in the District of Strumica (Macedonia)" in Man, vol. 54, pp. 11-13.

Gell Alfred, 1998, Art and Agency: An Anthropological Theory, Oxford University Press, Oxford, $296 \mathrm{p}$.

Gokalp Altan, 1995, «Le règne de l'écriture pour oreilles averties » in Revue des Mondes musulmans et de la Méditerranée, $\mathrm{n}^{\circ}$ 75-76, p. 19-28.

Gradeva Rossitsa \& Kuneralp Sinan, in press, "On Love, Religion, and Politics: Salonica (1876) and Ruse (1910)" in Gradeva Rossitsa (ed.), The Balkan Provinces of the Ottoman Empire: The Personal Dimension. I. Agents of Faith, Isis, Istanbul.

Hanganu Gabriel, 2010, "Eastern Christians and Religious Objects. Personal and Material Biographies entangled" in Hann Chris \& Goltz Hermann (eds.), Eastern Christians in Anthropological Perspective, University of California Press, Berkeley, pp. 33-55.

Herzfeld Michael, 1990, "Icons and Identity: Religious Orthodoxy and Social Practice in Rural Crete" in Anthropological Quarterly, vol. 63/3, pp. 109-121.

Herzfeld Michael, 1997, Cultural Intimacy. Social Poetics of the Nation-State, Routledge, New York, 226 p.

Hirschon Renée, 1989, Heirs of the Greek Catastrophe. The Social Life of Asia Minor Refugees in Piraeus, Clarendon Press, Oxford, $280 \mathrm{p}$.

Hopkin David, 2010, « Paul Sébillot et les légendes locales: des sources pour une histoire démocratiqué ? » in Bérose, Encyclopédie internationale des histoires de l'anthropologie, Paris, http://www.berose.fr/article478.html (consulté le 27/09/2019). 


\section{CAHIERS DE LITTÉRATURE ORALE}

52 Éclats de paroles $-\mathrm{n}^{\circ} 85$

IsnART Cyril, 2016, « Raconter saint Antoine à Lisbonne. Acteurs, performances et effets du discours hagiographique contemporain $\gg$ in Cahiers de littérature orale, $\mathrm{n}^{\circ} 79$, p. 95-122, https://doi.org/10.4000/clo.2656.

Kančov Vasil, 1996 [1900], Македония: етнография и статистика [La Macédoine : ethnographie et statistiques], Éditions Prof. Marin Drinov ( $1^{\mathrm{e}}$ édition, BKD), Sofia, $341 \mathrm{p}$.

Kenna Margaret, 1985, "Icons in Theory and Practice: An Orthodox Christian Example" in History of Religions (An International Journal for Comparative Historical Studies; Chicago), vol. 24/4, pp. 345-368.

Kopytoff Igor, 1986, “The Cultural Biography of Things. Commoditization as Process" in Appadurai A. (ed), The Social Life of Things, Cambridge University Press, Cambridge, pp. 64-91.

Krstić Tijana, 2011, Contested Conversions to Islam. Narratives of Religious Change in Early Modern Ottoman Empire, Stanford University Press, Stanford, $280 \mathrm{p}$.

Mesnil Marianne \& Popova Assia, 1993, « Démone et chrétienne : sainte Vendredi » in Revue des études slaves, vol. 65, $\mathrm{n}^{\circ}$ 4, p. 743-762, http:// www.jstor.org/stable/43270103.

Mondzain Marie-Josée, 1996, Image, icône, économie. Les sources byzantines de l'imaginaire contemporain, Seuil, Paris, $304 \mathrm{p}$.

Neveu Norig, 2016, « Les récits hagiographiques : lieux de mémoire et incarnation de l'esprit de résistance à Ma'ân (Jordanie) » in Cabiers de littérature orale, $\mathrm{n}^{\circ} 79$, p. 123-146, https://doi.org/10.4000/clo.2668.

Popović Alexandre \& Veinstein Gilles (dir.), 1995, Bektachiyya: études sur l'ordre mystique des Bektachis et les groupes relevant de Hadji Bektach, Isis Press, Istanbul, $476 \mathrm{p}$.

Rhew воттом David, 1975, “The Saint's Feast and Skopska Crna Goran Social Structure" in Man (N.S.), $\mathrm{n}^{\circ} 11$, pp. 18-34. 
LES « BIOGRAPHIES » D'UNE ICÔNE VOLANTE ET LE STATUT DE QUELQUES RÉCITS DANS UNE RÉGION BALKANIQUE

Sant-Cassia Paul, 2018, "Marriages at the Margins: Interfaith Marriages in the Mediterranean" in Journal of Mediterranean Studies, vol. 27/2, pp. 111-132.

STEWART Charles, 2008, "Dreaming of Buried Icons in the Kingdom of Greece" in Mazower Mark (ed.), Networks of Power in Modern Greece. Essays in Honor of John Campbell, Hurst \& Co, London, pp. 89-108.

STEWART Charles, 2012, Dreaming and Historical Consciousness in Island Greece, Harvard University Press, Cambridge, 278 p.

TAussig Michael, 1987, Shamanism, Colonialism and the Wild Man. A Study in Terror and Healing, The University of Chicago Press, Chicago/London, 517 p.

Trifunoski Jovan, 1979, Strumički kraj. Narodni život i običaji [Région de Strumica : vie et coutumes populaires], Akademija N.U, Skopje, 164 p.

Valtchinova Galia, 2006, Балжански ясновидки и пророчици през ХХ-ти век [Prophétesses et clairvoyantes des Balkans au $\mathrm{xx}^{\mathrm{e}}$ siècle], Éditions de l'Université de Sofia St. Kliment Okhridski, Sofia, 416 p.

Valtchinova Galia, 2019, Saints, Places and National Imagination. Historical Anthropology of Religiou Life in the Balkans, Isis Press, Istanbul, 273 p.

VRažInovski Tanas, 1999, Narodna tradicija. Religija-kultura [La tradition populaire. Religion-culture], Matica Makedonska, Skopje. 277 p.

Résumé : L'article est consacré à l'usage de l'icône orthodoxe en tant que support identitaire, par le prisme de quelques récits autour d'une icône réputée à miracles : l'icône « volante » du village de Konče, en République de Macédoine du Nord. L'analyse met en œuvre les notions d'agentivité et de biographie d'image, saisies à travers des récits. La reconstitution de la «biographie » de l'icône-qui-vole en question à travers des récits livrés par divers interlocuteurs, dans une succession d'études sur le terrain, est au cœur de l'article. Une attention particulière est accordée au statut des récits au regard des différents acteurs locaux qui les ont produits lors de mon entrée sur le terrain et à chaque « retour », sur plus d'une décennie. Entre la voyante octogénaire, ses descendants et proches, les femmes d'un cercle orthodoxe qu'elle a animé, des habitants anonymes de deux villages, un homme d'Église et les folkloristes locaux, les récits concernant 
cette icône ne cessent d'être réélaborés et requalifiés, changeant de contenu comme de statut. La partie conclusive est dédiée au décryptage de processus sociopolitiques plus vastes qui se dessinent en filigrane et dont l'identification permet de comprendre la portée de ces récits comme support d'une identité complexe et fragile qui s'est construite autour d'une série d'oppositions.

Mots-clefs : christianisme (orthodoxe), conversion, Grèce, icône, identité, islam, Macédoine, miracle, récits, réfugiés

\section{"Biographies" of a Flying Icon and the Status of a Few Narratives in a Balkan Region}

Abstract: The paper deals with the use of orthodox icons as symbolic device sustaining fragile identities. By focusing on the narrative settings of the stories of a miracle-working icon collected in the southeastern part of the Republic of Macedonia, the author provides an analysis in terms of agency and social biography of religious images. The paper scrutinizes the variety of contexts in which the story of the flying icon was reported on the turn of the $21^{\text {th }}$ century in several places, tracing back the appropriations and adaptations of what turned to be a migrant legend mainly attached to the village of Konce. The biography of the icon is reconstructed through the narratives produced by a variety of actors: a visionary woman, her family and mainly female network, anonymous inhabitants of two villages, a high-ranked servant of the Orthodox Church, and Macedonian folklorists. Particular attention is given to the status of the narratives, which varies from "history" of a village to a "legend" told with a sense of distance, to a neutral "report." The final section of the paper is dedicated to the wider sociopolitical processes underlying the story of the flying icon. They help understanding the narratives and the very act of narrating as a means for sustaining fragile collective identities.

Keywords: Christian (Eastern-Orthodox), conversion, Greece, icon, Islam, Macedonia, miracle, narrative, refugees

Galia Valtchinova est ethnologue, professeur d'anthropologie et spécialiste d'anthropologie historique des sociétés balkaniques. Elle a travaillé sur les aspects identitaires des cultes de saints, sur les femmes voyantes et prophétesses dans les cultures chrétiennes-orthodoxes des Balkans, ainsi que sur les pèlerinages et la mixité religieuse (dernier ouvrage : Saints, places and national imagination, Isis Press, Istanbul, 2019). 